\title{
Análisis bibliométrico de la Revista Española de Documentación Científica desde su inclusión en la Web of Science (2008-2018)
}

\author{
Jesús Cascón-Katchadourian*, Jose A. Moral-Munoz**, Huchang Liao***, Manuel J. Cobo**** \\ * Departamento de Información y Comunicación, Universidad de Granada \\ Correo-e: cascon@ugr.es | ORCID iD: https://orcid.org/0000-0002-3388-7862 \\ ** Departamento de Enfermería y Fisioterapia, Universidad de Cádiz. Instituto de Investigación e Innovación Biomédica de \\ Cádiz (INiBICA), Universidad de Cádiz \\ Correo-e: joseantonio.moral@uca.es | ORCID iD: https://orcid.org/0000-0002-6465-982X \\ *** Business School, Sichuan University \\ Correo-e: liaohuchang@163.com | ORCID iD: https://orcid.org/0000-0001-8278-3384 \\ **** Departamento de Ingeniería Informática, Universidad de Cádiz \\ Correo-e: manueljesus.cobo@uca.es | ORCID iD: https://orcid.org/0000-0001-6575-803X
}

Recibido: 28-05-2019; 2a versión: 26-09-2019; Aceptado: 11-11-2019.

Cómo citar este artículo/Citation: Cascón-Katchadourian, J.; Moral-Munoz, J. A.; Liao, H.; Cobo, M. J. (2020). Análisis bibliométrico de la Revista Española de Documentación Científica desde su inclusión en la Web of Science (2008-2018). Revista Española de Documentación Científica, 43 (3), e267. https://doi.org/10.3989/redc.2020.3.1690

\begin{abstract}
Resumen: En este trabajo se presenta un análisis bibliométrico del contenido científico de la Revista Española de Documentación Científica durante el periodo 2008-2018, usando como metodología el análisis de rendimiento y los mapas de la ciencia. En los resultados se muestran, por un lado, algunos de los indicadores bibliométricos de rendimiento básicos, como son: número de documentos publicados, citas obtenidas, evolución del Factor de Impacto, Índice-h, así como los autores más productivos y más citados y la distribución geográfica de las publicaciones. Por otro lado, este análisis muestra la evolución conceptual de la revista basada en los mapas de la ciencia. En la discusión se comparan estos resultados con otros estudios anteriores, que existen sobre la revista, de evolución temática, y sobre la documentación a nivel nacional e internacional. Se concluye indicando cuales son los autores, países y las dos grandes áreas temáticas que destacan en esta revista: bibliometría y web.
\end{abstract}

Palabras clave: Bibliometría; mapas de la ciencia; análisis de co-palabras; Índice-h; Web of Science; análisis de revistas; REDC.

\section{Bibliometric analysis of the Revista Española de Documentación Científica since its inclu- sion in the Web of Science (2008-2018)}

Abstract: In this work a bibliometric analysis of the scientific content of the Revista Española de Documentación Científica during the period 2008-2018 is presented, using as the methodology the performance analysis and the science mapping. The results show, on one hand, some of the basic bibliometric performance indicators, such as: number of documents published, citations received, evolution of the Impact Factor and h-index, as well as the most productive authors, most cited items and the geographical distribution of publications. On the other hand, this analysis shows the conceptual evolution of the journal, based on science mapping. In the discussion, those results were compared with other previous studies of thematic evolution that exist about this journal and about documentation both at the national and international level. We conclude indicating the authors, countries and the two major thematic areas that stand out in this journal: bibliometrics and web studies.

Keywords: Bibliometrics; science mapping; co-word analysis; h-index; Web of Science; journal analysis; REDC.

Copyright: (c) 2020 CSIC. Este es un artículo de acceso abierto distribuido bajo los términos de la licencia de uso y distribución Creative Commons Reconocimiento 4.0 Internacional (CC BY 4.0). 


\section{INTRODUCCIÓN}

La bibliometría es una herramienta útil para evaluar y analizar el resultado de la investigación académica. Está demostrado que contribuye al progreso de la ciencia de diversas maneras: permite evaluar el progreso realizado, identifica las fuentes más confiables de publicaciones científicas, identifica los principales actores científicos, sienta las bases académicas para la evaluación de nuevos desarrollos, etc.

Uno de los principales aportes de la bibliometría es el uso de criterios objetivos para poder evaluar la investigación desarrollada por investigadores, siendo cada vez más valorada como una herramienta para la medición de la calidad y productividad académica (Moed y otros, 1995). En definitiva, es un importante enfoque para evaluar y analizar la investigación desarrollada por diferentes actores, tal como grupos de investigación, centros de investigación, universidades, países, revistas y, en general, científicos.

En este sentido se suelen utilizar dos métodos principales para explorar un campo de investigación: el análisis del rendimiento y los mapas de la ciencia (Noyons y otros, 1999; Van-Raan, 2004). El objetivo del análisis de rendimiento es evaluar el impacto de la producción científica de diferentes actores científicos. Por otro lado, los mapas de la ciencia, sin embargo, tienen como objetivo mostrar la estructura conceptual, social o intelectual de la investigación científica así como su evolución y aspectos dinámicos.

Teniendo en cuenta la literatura disponible, son varios los autores que han analizado la trayectoria de la Revista Española de Documentación Científica (REDC) desde distintos puntos de vista. Algunos autores hacen un análisis sobre el área temática de Biblioteconomía y Documentación, y en dicho análisis se incluye y destaca la REDC: Frías Montoya (1996) hace una descripción de las Jornades sobre la Investigació en Biblioteconomia $i$ Documentació a Espanya, donde se menciona a la REDC como una de las más productivas; De-MoyaAnegón y Jiménez-Contreras (1999) analizan los autores españoles más citados en esta disciplina, donde la REDC aparece como la de mayor índice de impacto; Delgado-López-Cózar (2000) hace un diagnóstico del área mencionando a la REDC como la más prestigiosa; Guallar y otros (2017), realizan un análisis temático y metodológico de las revistas científicas del área, entre ellas la REDC, analizando los artículos desde el 2012 al 2014; Ferran-Ferrer y otros (2017) publican un artículo con el mismo marco temporal que analiza los métodos y técnicas de investigación.
Por otra parte, también hay artículos que analizan, en exclusiva, la trayectoria de la revista desde diversos aspectos: Pérez-Álvarez-Ossorio (1997) describe la cobertura temática de la revista en sus primeros 20 años, basándose en los índices acumulativos de la misma; Ríos-Hilario (2001) analiza los métodos de investigación en la revista desde 1989 a 1999: temática, metodología, población, muestra, etc.; Jiménez-Hidalgo (2007) investiga sobre la autoría de los artículos de la REDC como una de las revistas de mayor calidad del área; el Equipo Editorial de la REDC (2012) publica un interesante editorial sobre la evolución histórica de la misma a los 36 años de la edición del primer número. Evolución que va paralela al desarrollo de la disciplina; Ríos-Hilario y TraviesoRodríguez (2013) actualizan el artículo de Ríos Hilario (2001) visto anteriormente, con la misma metodología, si exceptuamos el análisis temático que ya no se realiza; Pandiella-Dominique y otros (2019) estudian el sexenio 2010-2015, que tiene como principales resultados las redes de citación de este ámbito en WoS y en la REDC, así como la especialización temática y la internacionalización de la revista.

De acuerdo con la literatura se han detectado algunos aspectos no estudiados hasta la actualidad. En primer lugar, faltan estudios cuyo marco temporal sea más actual. De todo lo expuesto anteriormente tan sólo los artículos de Guallar y otros (2017), Ferran-Ferrer y otros (2017) y PandiellaDominique y otros (2019) presentan estudios de años más cercanos. A pesar de ello, el último año analizado es el 2015, en el mejor de los casos. De estos artículos sólo el de Pandiella-Dominique y otros (2019) se centra en la REDC.

En segundo lugar, aunque varios de los artículos anteriores analizan conceptos de los temas de investigación, estos se han realizado basándose en los índices acumulativos de la revista (Pérez-ÁlvarezOssorio, 1997) o por medio del análisis de contenido realizado por especialistas en la materia (PandiellaDominique y otros, 2019). No hay ningún estudio que utilice la metodología aquí presentada para analizar los temas de investigación tratados en la REDC. En nuestra opinión, es interesante observar si coinciden o no en sus resultados con los anteriores estudios. También consideramos que, a la hora de clasificar los documentos de una revista, esta metodología es más fácilmente reproducible, puesto que no se basa en lo que consideran las personas, sino en los datos de una base de datos, prueba de ello es el número de artículos publicados en los últimos años en los que se analizan revistas a través de Science Mapping con SciMAT (López-Robles y otros, 2019) (Cobo y otros, 2015b), con otros softwares disponibles (Merigó y otros, 2019) o bien analizando áreas de conocimiento (Moral-Munoz y otros, 2019). 
En este sentido el artículo que se propone se diferencia de los anteriores, ya mencionados, por varios motivos: por el marco temporal que analiza, los últimos 11 años (2008-2018) que son los que lleva la revista siendo indexada en la Web of Science (WoS), por tanto, también es un artículo a larga escala. Además, se diferencia por el análisis de los temas de investigación y su evolución conceptual a lo largo del periodo, todo ello usando las herramientas de los mapas de la ciencia que nos proporciona SciMAT (Cobo y otros, 2012a). Por tanto, el objetivo principal de este artículo es llevar a cabo un análisis bibliométrico de la investigación realizada por la Revista Española de Documentación Científica desde su inclusión en la WoS en el año 2008 hasta la actualidad. El presente estudio persigue analizar el rendimiento científico de la REDC desde su inclusión en la WoS, así como el análisis de las temáticas y su evolución a través de las técnicas de generación de mapas científicos, pretendiendo no una categorización de los artículos creando categorías genéricas, sino una descripción más profunda de las temáticas.

En esta investigación, por un lado, se realiza un análisis bibliométrico del rendimiento de la revista mostrando datos sobre algunos indicadores importantes y, por otro lado, se analiza la evolución temática a través de mapas de la ciencia. Este artículo está estructurado de la siguiente manera: en el segundo apartado, se presenta la metodología. En el tercero, se evalúa la revista con un análisis bibliométrico del rendimiento a lo largo del marco temporal ya expuesto. En el cuarto, se presenta el análisis de los mapas de la ciencia de la REDC. Finalmente, se extraen algunas conclusiones en el quinto apartado.

\section{METODOLOGÍA}

En primer lugar, para llevar a cabo el análisis del rendimiento y los mapas de la ciencia, hay que recopilar los documentos científicos publicados por la REDC. Los documentos se han descargado desde Web of Science de Clarivate (WoS). Para ello, se utilizó la búsqueda avanzada con la siguiente ecuación de búsqueda: SO=(REVISTA ESPANOLA DE DOCUMENTACION CIENTIFICA). Esta búsqueda recuperó un total de 399 documentos desde el año 2008 hasta el año 2018, ambos inclusive. Fue filtrada por tipo de documento (ARTÍCULOS O REVISIONES) obteniendo un total de 337 documentos en dicho marco temporal. Se han utilizado también las citas recibidas por dichos documentos en ese tiempo; los datos fueron descargados el 04/02/2019 cuando la WoS estaba actualizada hasta el día 01/02/2019.
En segundo lugar, se realizó el análisis basado en indicadores bibliométricos de rendimiento, para lo cual se utilizaron los siguientes indicadores: documentos publicados, citas recibidas, Factor de Impacto de la revista (Garfield, 1972), Índice-h de la revista (Alonso y otros, 2009; Hirsch, 2005), autores más citados, y los datos sobre la distribución geográfica de las publicaciones.

El Factor de Impacto de la REDC lo publica Clarivate Analytics a través de su producto InCites Journal Citation Reports (JCR). Como el Factor de Impacto se calcula teniendo en cuenta los dos años anteriores, y la revista entró en la WoS en el año 2008, el primer año disponible es el 2010 y el último factor de impacto disponible a la fecha de la realización de este estudio es el del año 2017.

El Índice-h es uno de los principales indicadores bibliométricos de la producción y la calidad científica; fue diseñado por Hirsch (Hirsch, 2005) para medir el rendimiento de los investigadores (Alonso y otros, 2009). En su definición original vemos cómo este indicador fue desarrollado en un primer momento para medir el rendimiento científico de los investigadores:

"Un científico tiene índice $h$ si el $h$ de sus $N p$ () trabajos recibe al menos $h$ citas cada uno, y los otros $(N p-h)$ trabajos tienen como máximo

$h$ citas cada uno".

\section{Siendo Np el número total de artículos.}

Sin embargo, este índice se ha utilizado también para medir el rendimiento de los diferentes actores científicos (Alonso y otros, 2009), como pueden ser las revistas (Braun y otros, 2006), países (Guan y Gao, 2008), y centros de investigación o universidades (Schubert, 2007). En este caso se va a utilizar para medir el rendimiento de la REDC para todo el periodo que estamos estudiando, desde 2008 hasta 2018.

En tercer lugar, se hicieron los mapas de la ciencia, que son representaciones espaciales de las relaciones que existen entre documentos, autores, campos o disciplinas. Se han utilizado ampliamente en la literatura científica en muy diversos campos para mostrar estas relaciones gráficamente (Cartes-Velásquez y Manterola Delgado, 2014; Huang y Chang, 2014; Liu y otros, 2012; Montero-Díaz y otros, 2018; Raeeszadeh y otros, 2018; Tang y Shapira, 2011)

Esos análisis de mapas de la ciencia se pueden realizar con diversas herramientas, programas informáticos (Cobo y otros, 2011b). En esta investigación hemos usado SciMAT (Science Mapping Analysis software Tool) (Cobo y otros, 2012a) puesto que integra todo lo que requerimos para esta investigación y, además, es gratuito y de software libre. 
Los documentos en bruto se descargaron de la WoS como texto sin formato incluyendo el registro completo. Este documento txt se introdujo en SciMAT para construir la base de datos de donde se generarán posteriormente los mapas de la ciencia. Para mejorar la calidad de los datos se aplicó un proceso de control de autoridades (las palabras clave de los autores y los ISI keywords plus se utilizaron como unidad de análisis). Igualmente, las palabras que representan los mismos conceptos se agruparon realizando un proceso similar. Además, algunas palabras clave sin sentido en este contexto, como las palabras vacías, se eliminaron. Por último, se preprocesaron los autores y las afiliaciones.

A continuación, utilizando el administrador de períodos de SciMAT, se establecieron dos períodos consecutivos de tiempo para mostrar la evolución conceptual de la REDC en el análisis de mapas de la ciencia. Para evitar la dispersión de los datos que impidiera su comparación, la mejor opción hubiera sido elegir períodos de un año. Sin embargo, no se generaron suficientes datos en el lapso de un año para obtener buenos resultados con el análisis de mapas de la ciencia. Por esta razón, el marco temporal original (2008-2018) se subdividió en dos periodos procurando que ambos periodos tuvieran el mismo número de documentos, y que abarcaran el mismo periodo de tiempo. Al ser nuestro marco temporal de 11 años, se tomó la decisión de considerar un primer período de seis años (2008-2013), y un segundo periodo de cinco años (2014-2018) como mejor manera de cumplir con los criterios antes mencionados. Los datos se han dividido, por tanto, en dos periodos de tiempo consecutivos con 170 y 167 documentos, respectivamente, y con 729 y 952 palabras clave.

Siguiendo la metodología de Cobo y otros (2011a) basada en un análisis de co-palabras (CaIlon y otros, 1983) y en el Índice-h (Hirsch, 2005), se consideran 4 fases para analizar un campo de investigación o revista:

1. Detección de los temas de investigación. Se aplica un análisis de co-palabras (Callon y otros, 1983) a los datos en bruto para cada periodo establecido sobre los documentos de la revista; a ello le añadimos un clustering de palabras clave usando el algoritmo del centro simple (Coulter y otros, 1998), que localiza redes de palabras clave que están muy vinculadas entre sí y que se corresponden con centros de interés o problemas de investigación de interés significativo entre los investigadores. Esta similitud entre las palabras clave se evalúa utilizando el índice de equivalencia o equivalence index (Coulter y otros, 1998): $e_{i j}=c^{2}{ }_{i j} / c_{i} C_{j}$, donde $c_{i j}$ es el número de documentos en los que coexisten dos palabras clave $i$ y $j$, y $c_{i}$ y $c_{j}$ representan el número de documentos en los que aparece cada una.

2. Visualización de temas de investigación y red temática. En la segunda fase, a través de dos instrumentos de visualización diferentes se visualizan los temas más destacados; se trata del diagrama estratégico $(\mathrm{He}, 1999)$ y la red temática. Cada uno de estos temas está caracterizado por dos medidas (Coulter y otros, 1998), la centralidad y la densidad. La centralidad mide el grado de interacción de una red con otras redes, se define cómo $c=10 * \Sigma e_{k h}$, donde $\mathrm{k}$ es una palabra clave que pertenece a ese mismo tema y $h$ una palabra clave que pertenece a otros temas.

Por otro lado, la densidad mide la intensidad interna de la red y se puede definir como $d=100\left(\Sigma e_{i j} / w\right)$, donde $i$ y $j$ son palabras clave que pertenecen al tema, y $w$ el número de palabras clave en el tema. Con estas dos medidas, podemos visualizar el campo de investigación de la revista como un conjunto de temas de investigación, lo cual podremos visualizar a través de un diagrama estratégico bidimensional (Figura 1 ) donde se clasifican los temas de investigación en cuatro grupos:

(a) En el cuadrante superior derecho se encuentran los temas que presentan una centralidad fuerte y una alta densidad; son, por tanto, los temas motores de la revista. Estos están bien desarrollados y son importantes para la estructura del campo de investigación o, en este caso, para la revista.

(b) En el cuadrante superior izquierdo se localizan los temas muy especializados pero periféricos, es decir, tienen una importancia marginal para el campo, ya que estos temas están internamente bien desarrollados pero sus enlaces con otros temas externos son muy débiles.

(c) En el cuadrante inferior izquierdo están representados los temas emergentes o en desaparición, puesto que tienen una densidad y centralidad bajas. Son temas poco desarrollados y marginales.

(d) En el cuadrante inferior derecho, por último, se presentan temas transversales y generales, es decir, básicos, son temas importantes para el campo de investigación o la revista, pero no están altamente desarrollados. 
Figura 1. Diagrama estratégico sobre campos o temas de investigación

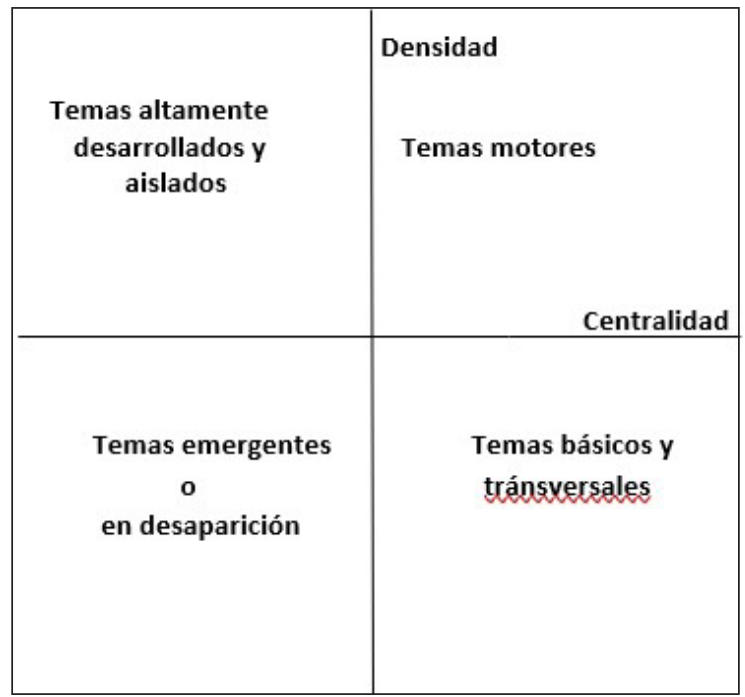

3. Descubrimiento de áreas temáticas. En esta tercera fase se detectan los temas de investigación a lo largo de un periodo de tiempo; posteriormente, se analizan las áreas generales de evolución de la revista, los orígenes de las mismas y sus interrelaciones. Para ello, se utiliza el índice de inclusión (Sternitzke y Bergmann, 2009) que detecta nexos conceptuales existentes entre temas de investigación en diferentes períodos. Con ello conseguimos identificar las áreas temáticas de un campo de investigación o de una revista. Se define área temática como un conjunto de temas que han evolucionado durante varios períodos de tiempo. Hay que decir que un tema de investigación particular puede pertenecer a un área temática, a varias, o a ninguna de las áreas temáticas identificadas; en este último caso se puede interpretar que este tema de investigación es el origen de una nueva área temática en la revista. Posteriormente se realiza un mapa bibliométrico con la evolución temática en dos periodos de tiempo, los temas pueden no estar vinculados, estar vinculados por líneas continuas o líneas de puntos. Además, según que el índice de inclusión sea más o menos grande, así será el grosor de las líneas que unen los temas vinculados (Cobo y otros, 2015b).

4. Análisis de rendimiento. En esta fase la contribución relativa de los temas de investigación y las áreas temáticas a todo el campo de investigación de la revista es medido, tanto cuantitativa como cualitativamente, para establecer cuáles son los subcampos con más importancia, producción y de mayor impacto. Realizamos el análisis de rendimiento como un complemento del trabajo de mapeo científico; para ello algunos de los indicadores bibliométricos utilizados son: número de documentos publicados, número de citas y diferentes tipos de Índice-h (Alonso y otros, 2009; Hirsch, 2005; Martínez y otros, 2014). Para evaluar estos indicadores de rendimiento, el programa, mediante una función, asigna un conjunto de documentos a cada tema detectado; en concreto, SciMAT devuelve la unión algebraica del conjunto de documentos asociados con las palabras clave del tema. Un documento tiene varias palabras clave, ya que cada una de ellas puede estar asociada a un tema diferente; un documento podría estar asociado a varios temas. Además, como las áreas temáticas podrían compartir algunos temas, también podrían compartir algunos documentos.

\section{RESULTADOS}

\section{1. Análisis bibliométrico del rendimiento de la REDC}

\subsubsection{Publicaciones y citas}

La distribución de publicaciones por año se muestra en la Figura 2. Como se puede observar, el número de publicaciones de la REDC se han mantenido en los últimos 11 años relativamente estable, habiéndose publicado 24 documentos el año que menos (2008), hasta 40 el año que más (2013). Como podemos ver en la Figura 2, el periodo 2008-2012 es estable en cuanto a publicaciones, en torno a los 26 documentos al año, de media, con una fuerte subida en el año 2013 llegando a los 40 documentos; posteriormente se produce un paulatino y ligero descenso en el número de documentos publicados desde ese año hasta la actualidad, para situarse en el 2018 ligeramente por encima del número de artículos que se publicaban en 2008-2012.

La distribución del recuento de citas logradas por esos documentos se muestra en la Figura 3. Se tienen en cuenta los datos tomados de la WoS directamente; por ejemplo, para el año 2018 se muestran las citas recibidas por la revista en ese año (de todos sus artículos publicados en todos los años), por el resto de las revistas indexadas en la WoS. Por tanto, esta medida nos muestra si una revista es citada más (interesa más) en unos años que en otros. Se pueden observar 2 periodos, ambos alcistas, el primero desde 2008 al 2014 y el segundo desde 2015 al 2018. En el primero se pasa de 1 sola cita hasta las 150 del año 2014, un aumento 
ciertamente espectacular, explicado, en parte, por haber sido indexada en la WoS. Destacar el aumento de citas en el año 2013 donde se producen el doble de citas que el año anterior. El segundo periodo empieza con una bajada de citas respecto al año 2014 de casi un tercio de las mismas (2014: 151 ; 2015: 108) pero desde ese año hasta 2018 el número de citas sube paulatinamente hasta superar al periodo anterior y alcanzar las 161.

Si ponemos en relación el número de documentos publicados con el número de citas recibidas, sobre todo desde el año 2014, observamos cómo, a pesar de haber un descenso del número de artículos publicados, hay un aumento del número de citas, lo que explica que a pesar de que se publique un número regular de artículos en esta revista, éstos van siendo cada vez más interesantes para la comunidad científica.

\subsubsection{Factor de Impacto}

En la Figura 4 podemos ver el factor de impacto desde el año 2010 hasta el 2017. Incluimos en el gráfico información relativa a la posición (cuartil) dentro de la categoría en la que se encuentra que es "Information Science \& Library Science".

La gráfica de columnas muestra un gráfico con forma de dientes de sierra que va ascendiendo poco a poco, lo cual se puede confirmar con la línea de tendencia que empieza en un factor de impacto de

Figura 2. Número de registros en la WoS por año de publicación de la REDC

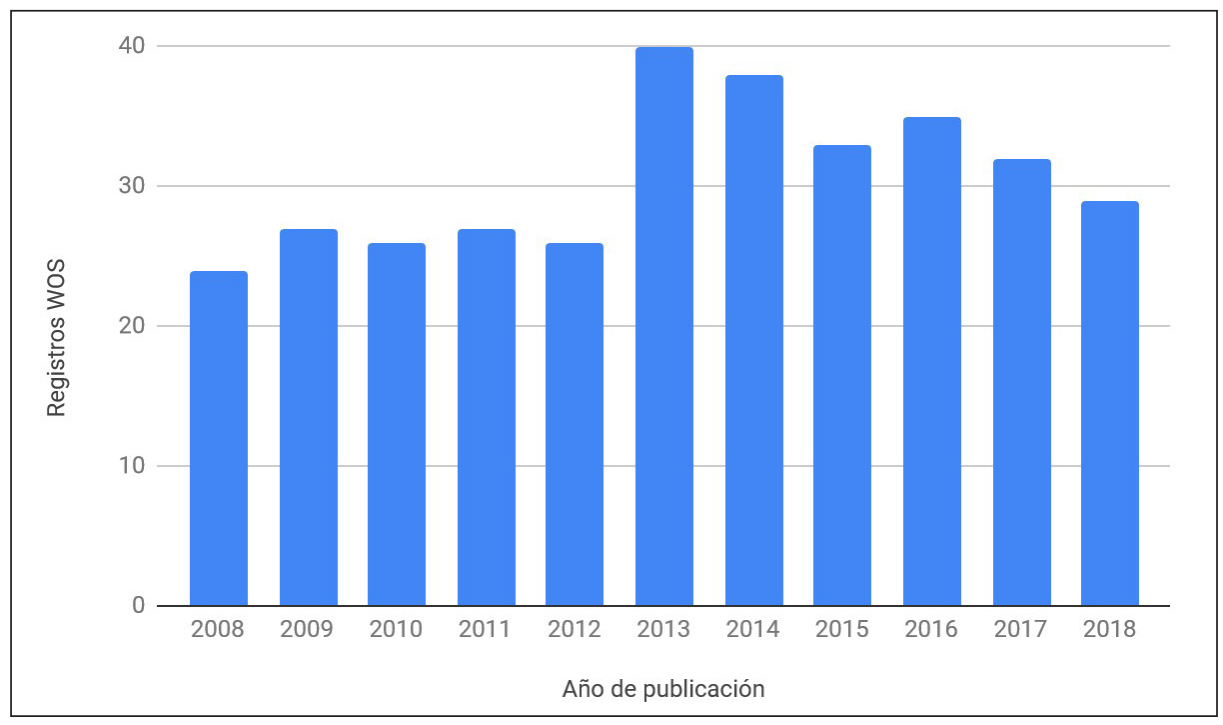

Figura 3. Número de citas recibidas por la REDC en cada año

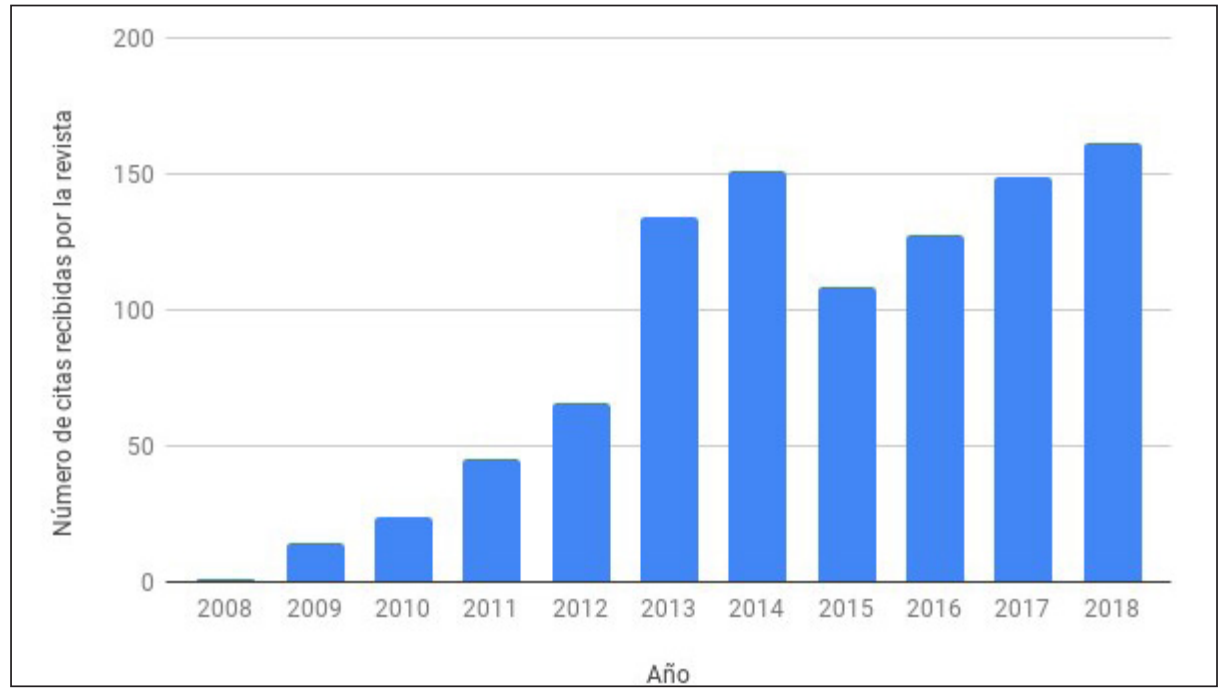


0,500 y llega a subir a 0,717. La REDC empieza en un Q3, en los años intermedios subió a un segundo cuartil y desde 2015 descendió al tercero. Aunque el número de revistas en la categoría "Information Science \& Library Science" ha subido desde el año 2010 al 2017, de 77 a 88 revistas, y eso puede influir en la posición en el ranking de las revistas consolidadas como la REDC, si observamos la media del factor de impacto de la categoría según el InCites Journal Citation Reports, en estos años ha subido desde 0,761 a 1,180. Ello quiere decir que, aunque el factor de impacto de la REDC sube ligeramente, el resto de las revistas de su categoría han subido ese factor de impacto más rápidamente.

En cuanto al Índice-h de la REDC, según la WoS, es 13.

\subsubsection{Autores más productivos y más citados y distribución geográfica de la publicación}

En las siguientes tablas (Tablas I y II) encontramos a los autores más productivos y a los más citados. En cuanto a los autores más productivos hay que destacar en los 3 primeros lugares a Jiménez-Contreras, López-Cózar y Torres-Salinas, señalando que, además de ser de nacionalidad española, los tres provienen de la Universidad de Granada. En cuanto a los autores más citados, tenemos a los mismos autores, salvo que cambian la posición: en segundo lugar, aparece Torres Salinas por delante de López-Cózar. Estos datos concuerdan con los que ofreció en su momento el Equipo Editorial REDC (2012) donde la Universidad de Granada destaca con el $13 \%$ de todos los artículos publicados por las universidades en esta revista.
En cuanto a la distribución geográfica de los artículos, podemos ver en la Tabla III cómo el país con más publicaciones en la REDC es España, con mucha diferencia; de hecho, ha publicado más artículos que el resto de los países juntos. En el top 3 tendríamos también a Cuba y Colombia. Hay que bajar al puesto 6 y 7, donde encontramos a Brasil y Portugal, para encontrar países no hispanohablantes. Por último, destacar que entre los 10 países que más artículos han publicado en la REDC, solo aparece Reino Unido como país no iberoamericano. Por tanto, la REDC tiene y recibe una clara influencia española en primer lugar e iberoamericana en segundo lugar (Tabla III). Hay que tener en cuenta que algunos artículos se han realizado en colaboración entre dos o más países, debido a ello, la sumatoria de los artículos de la Tabla III (356) es mayor que el número de artículos analizado en este estudio (337).

\subsection{Análisis de los mapas de la ciencia de REDC}

\subsubsection{Análisis del contenido de los trabajos publicados}

El análisis se realiza por periodos; se irán apoyando las afirmaciones con las tablas y diagramas estratégicos que mostrarán los temas más destacados de la revista. En los diagramas estratégicos el tamaño de la esfera de cada tema de investigación es proporcional al número de documentos publicados en dicho tema, además, se añade entre paréntesis el número de citas obtenidas por cada tema de investigación.

Figura 4. Factor de impacto de la REDC por año

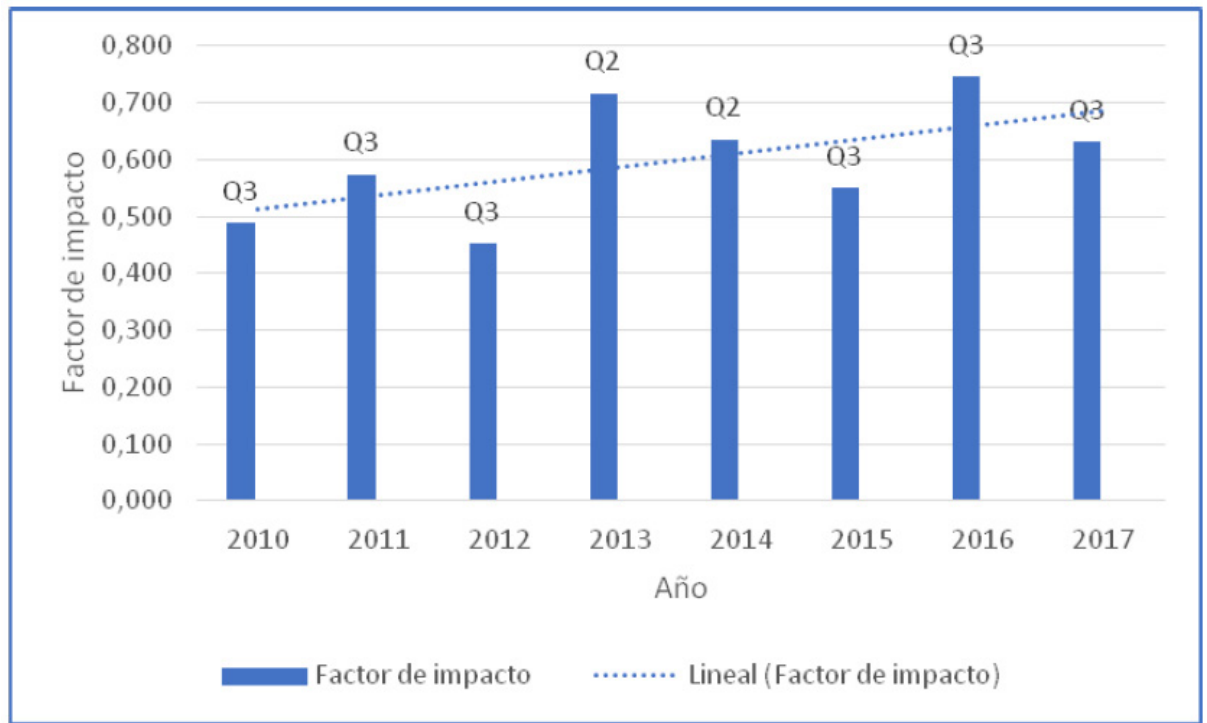


Tabla I. Autores más productivos

\begin{tabular}{|l|c|}
\hline Nombre & $\begin{array}{c}\text { Número de } \\
\text { documentos }\end{array}$ \\
\hline JIMENEZ-CONTRERAS, E & 11 \\
\hline LOPEZ-COZAR, ED & 9 \\
\hline TORRES-SALINAS, D & 9 \\
\hline BORDONS, M & 7 \\
\hline ALEIXANDRE-BENAVENT, R & 6 \\
\hline ORDUNA-MALEA, E & 6 \\
\hline MORILLO, F & 5 \\
\hline OSCA-LLUCH, J & 5 \\
\hline ROBINSON-GARCIA, N & 5 \\
\hline SANZ-CASADO E & 5 \\
\hline
\end{tabular}

Tabla II. Autores de la REDC más citados

\begin{tabular}{|l|c|}
\hline Nombre & Citas recibidas \\
\hline JIMENEZ-CONTRERAS, E & 80 \\
\hline TORRES-SALINAS, D & 75 \\
\hline DELGADO LOPEZ-COZAR, ED & 61 \\
\hline ROBINSON-GARCIA, N & 44 \\
\hline BORDONS, M & 33 \\
\hline GONZALEZ ALCAIDE, G & 29 \\
\hline ORDUNA-MALEA, E & 27 \\
\hline VALDERRAMA ZURIAN, JC & 23 \\
\hline MORILLO, F & 23 \\
\hline FERNANDEZ-QUIJADA, D & 18 \\
\hline DE FILIPPO, D & 18 \\
\hline DORTA-GONZALEZ, P & 18 \\
\hline MARTIN-MARTIN, A & 18 \\
\hline SANZ-CASADO, E & 18 \\
\hline
\end{tabular}

Primer período (2008-2013). Como se puede ver en la Figura 5, en este primer periodo, la REDC gira sobre 8 temas de investigación, fundamentalmente: Bibliometrics, Bibliometrics-indicators, Indicators, Web, Repositories, Productivity, Growth, y Efficiency. En la Tabla IV podemos ver los indicadores de rendimiento: número de documentos, citas obtenidas por esos documentos y el Índiceh. De acuerdo con esos indicadores, podríamos destacar los tres temas principales: Bibliometrics, Bibliometrics-indicators e Indicators. El impacto de estos tres temas es muy alto, sumando casi 1000 citas entre los tres, mientras que todos los demás
Tabla III. Distribución geográfica de los artículos de la REDC

\begin{tabular}{|l|c|}
\hline País & Número de artículos \\
\hline ESPAÑA & 288 \\
\hline CUBA & 17 \\
\hline COLOMBIA & 11 \\
\hline MEXICO & 9 \\
\hline ARGENTINA & 8 \\
\hline BRASIL & 7 \\
\hline PORTUGAL & 5 \\
\hline CHILE & 4 \\
\hline PERÚ & 4 \\
\hline INGLATERRA & 3 \\
\hline
\end{tabular}

temas juntos no llegan a 300 citas. El Índice-h de estos temas también es más alto, aunque no se observa una diferencia tan grande como en el número de citas. Por otro lado, y a gran distancia, tenemos dos temas más con cierta relevancia: Web y Repositories, con 21 y 12 documentos; ambos tienen un número de citas muy similar: 78 citas de Repositories y 76 de Web, es decir, con menos documentos obtiene más citas y un Índice-h superior.

El tema Bibliometrics-Indicators es un tema motor; a pesar de no ser el tema con el mayor número de documentos, sí es el que tiene más citas en este periodo y el que tiene un Índice-h más alto. Se relaciona con indicadores bibliométricos como el Índice-h, citation-impact y la co-autoría, pero también está muy relacionado con la evaluación de revistas, de universidades y con la investigación científica. Estos indicadores bibliométricos están relacionados también con la Web of Science, particularmente orientados a las Ciencias Sociales y al Social-Sciences-Citation-Index.

El otro tema motor destacado es Indicators; con solo 5 documentos menos que el anterior (47-52), tiene una tasa de impacto bastante menor, con casi la mitad de las citas que Bibliometrics-Indicators. Aunque pueda parecer que ambos temas están relacionados, Indicators engloba indicadores de más amplio espectro como pueden ser indicadores de calidad, de evaluación, innovación, sobre redes, webometrics, etc, es decir indicadores de más amplio espectro que los propiamente obtenidos por técnicas bibliométricas. Estos indicadores pueden evaluar la ciencia en general, la investigación o el ámbito de la revista que es Information-Science, así como analizar las redes sociales o la colaboración científica. 
Figura 5. Diagrama estratégico de la REDC del periodo 2008-2013

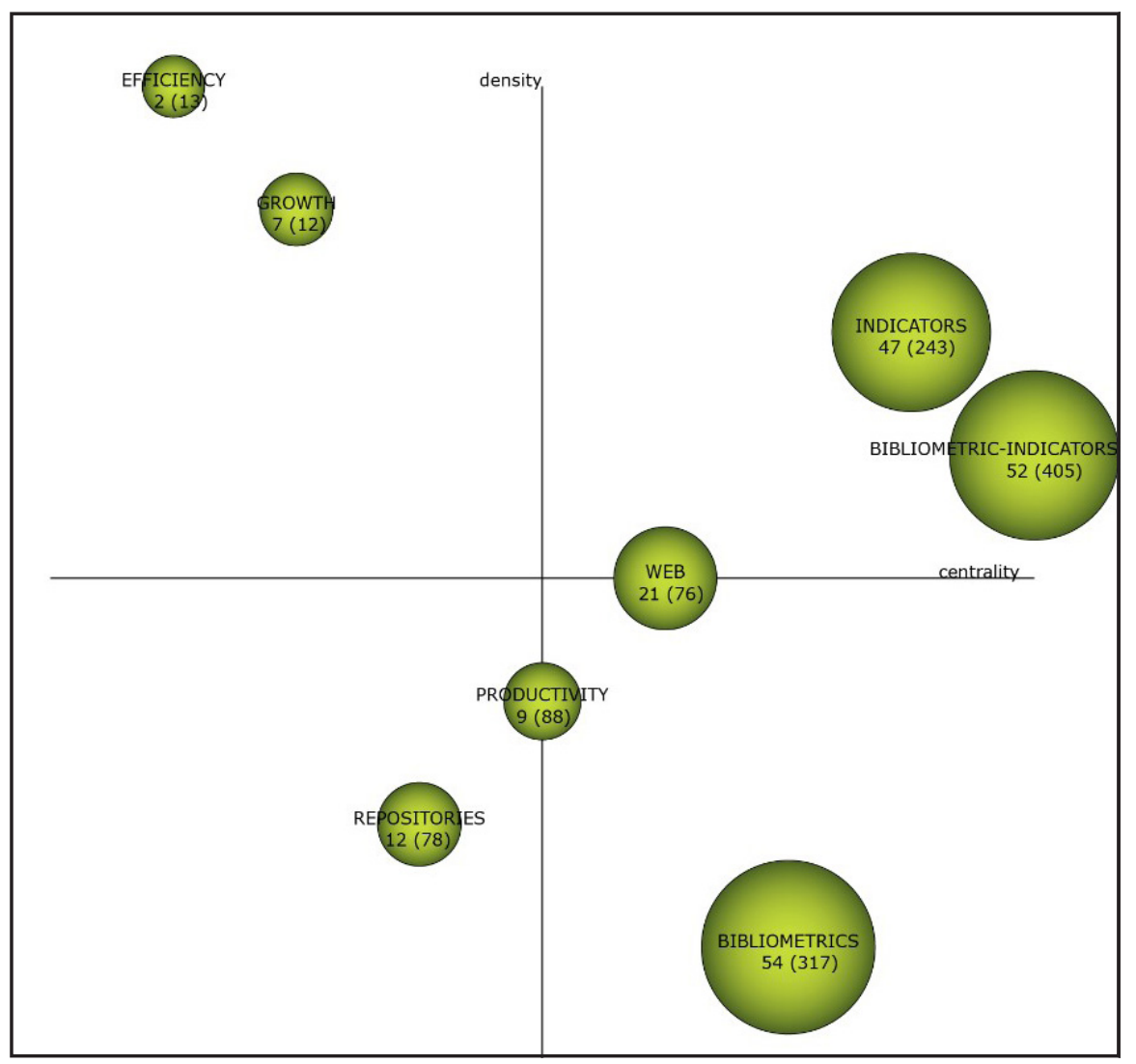

El tercer tema importante, básico y transversal de la revista, es la Bibliometría; se trata de la temática sobre la que tratan mayor número de documentos en este periodo, el segundo en citas y en Índice-h, por encima, en ambos casos, de Indicators. Está relacionado con Bibliometric-Indicators, pero es más general que éste (por ello es básico y transversal) ya que, aunque contiene un indicador tan importante como el Impact Factor, abarca el estudio y análisis de la producción científica de los profesores, grupos de investigación y las revistas científicas, así como las tendencias, las colaboraciones y las redes de coautoría.

El tema Web está en la encrucijada entre los temas motores y los transversales y básicos; está bastante lejos en importancia de los tres ya señalados, tanto en número de documentos como en Índice-h representa cuantitativamente menos de la mitad, y en citas tiene la cuarta parte que tienen los temas anteriormente referidos. Comprende temas como accesibilidad, usabilidad o análisis de contenido, además de los obvios como es Internet.

El tema Repositories es emergente, poco especializado; es en número de documentos inferior a
Web, pero es algo superior en citas y en Índice-h; aun así, está alejado de todos los ya analizados. Este tema está formado por Open-Access y Scientific-Journal, por lo que está relacionado con repositorios académicos de acceso abierto.

Por último, en este periodo tenemos los temas Crecimiento y Eficiencia. El tema Crecimiento está relacionado con el mundo de las patentes y con la tecnología (Figura 6), es decir se refiere al crecimiento tecnológico e industrial y a su producto documental que son las patentes. Por otro lado, el tema Eficiencia tiene que ver con los sistemas de indicadores que tratan de analizar la eficiencia, sobre todo de las instituciones universitarias.

Segundo periodo (2014-2018). En este segundo periodo la investigación gira en torno a 10 temas como se puede ver en la Figura 7, de los cuales, 6 son los principales si tenemos en cuenta los motores y los básicos: University-Libraries, Innovation, Scientific-Collaboration, Impact-Factor, ScientificProduction y Web. En comparación con el periodo anterior, en el segundo periodo hay 10 temas en lugar de 8, esta diversificación influye en los indicadores de rendimiento. 
Figura 6. Diagrama estratégico del tema crecimiento en el periodo 2008-2013 de la REDC

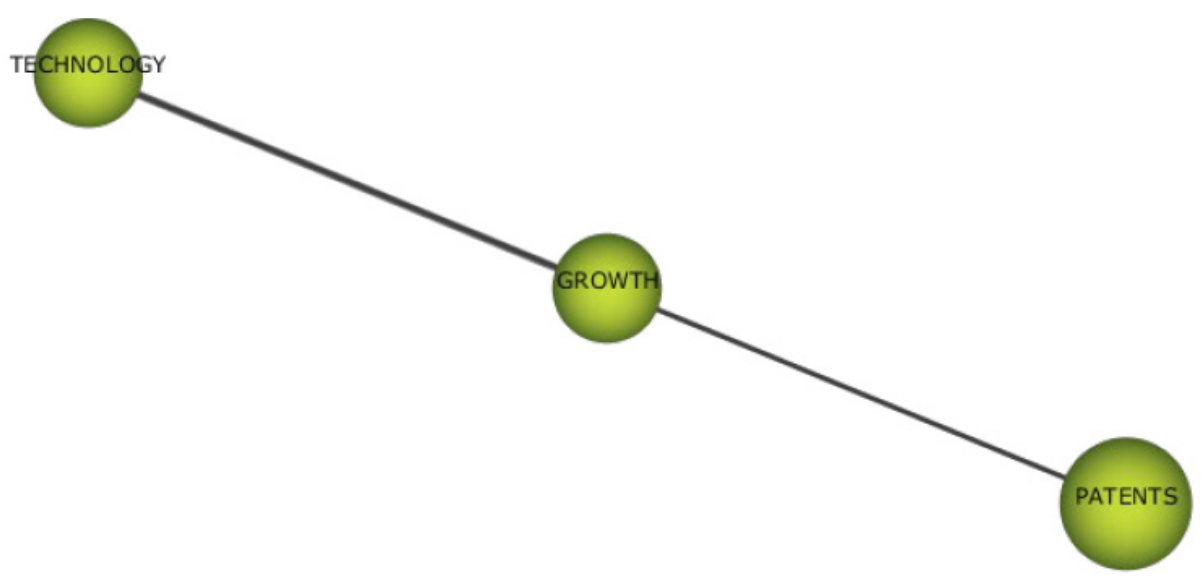

Tabla IV. Rendimiento de los temas de la REDC en el periodo 2008-2013

\begin{tabular}{|l|c|c|c|}
\hline Nombre & $\begin{array}{c}\text { Número de } \\
\text { documentos }\end{array}$ & Índice-H & $\begin{array}{c}\text { Número de } \\
\text { citas }\end{array}$ \\
\hline BIBLIOMETRICS & 54 & 11 & 317 \\
\hline BIBLIOMETRICS-INDICATORS & 52 & 12 & 405 \\
\hline INDICATORS & 47 & 8 & 243 \\
\hline WEB & 21 & 5 & 76 \\
\hline REPOSITORIES & 12 & 6 & 78 \\
\hline PRODUCTIVITY & 9 & 5 & 88 \\
\hline GROWTH & 7 & 3 & 12 \\
\hline EFFICIENCY & 2 & 2 & 13 \\
\hline
\end{tabular}

Si observamos dichos indicadores de rendimiento de la Tabla $V$ en cuanto al Índice-h hay que destacar que el nivel máximo es 6 y después 4 . En cuanto a las citas destacan los temas Impact-Factor, Scientific-Production y Scientific-Collaboration, con 86,59 y 35 citas respectivamente.

De los 6 temas destacados, se observa en la Tabla $\mathrm{V}$ cómo vertebran el campo de investigación de la revista, poniendo en relación el número de documentos, el Índice-h y el número de citas, los siguientes 4: Scientific-Production, Impact-Factor, Web y Scientific-Collaboration.

El tema Impact Factor es un tema motor, está casi en un término medio entre mayor y menor densidad; sin embargo, es el tema con mayor centralidad de todos al estar lo más a la derecha posible en el cuadrante; es, por tanto, un tema muy bien enlazado con el resto. Es el que mayor número de citas y mayor Índice-h tiene, siendo el segundo con más documentos de este periodo. Es la evolución del tema del periodo anterior, más amplio, denominado indicadores. Por su gran centralidad este cluster contiene otras temáticas amplias y de gran tamaño como Open-Access o las revistas en general, pero también con otros más concretos como las revistas académicas, las revistas científicas, los indicadores como el Índice-h u otros de colaboración internacional, así como bases de datos como Scopus.

El tema Scientific-Collaboration es un tema motor con una buena densidad y centralidad, posee el segundo mejor Índice-h de este periodo gracias a ser el quinto en número de documentos, pero el tercero en número de citas. Las temáticas que engloba figuraban, en parte anteriormente, en el cluster de Bibliometría. Contiene en su interior, como es obvio, conceptos como colaboración, investigación colaborativa y redes, pero también está relacionado con conceptos como conocimiento, productividad y Big-Science. 
Figura 7. Diagrama estratégico del periodo 2014-2018 de la REDC

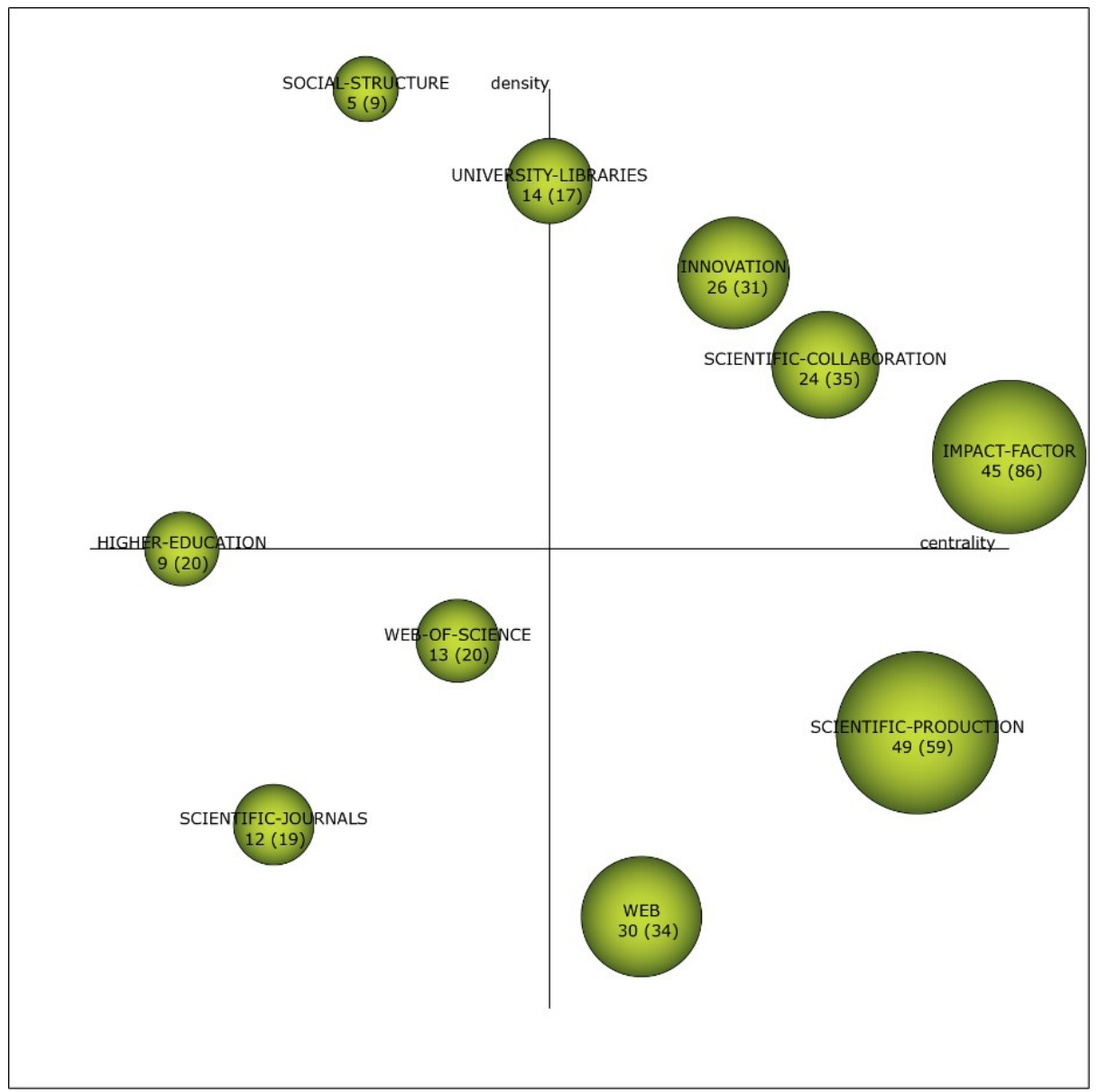

Tabla V. Rendimiento de los temas de la REDC en el periodo 2014-2018

\begin{tabular}{|l|c|c|c|}
\hline Nombre & Número de documentos & H- Index & Número de citas \\
\hline SCIENTIFIC-PRODUCTION & 49 & 4 & 59 \\
\hline IMPACT-FACTOR & 45 & 6 & 86 \\
\hline WEB & 30 & 3 & 34 \\
\hline INNOVATION & 26 & 3 & 31 \\
\hline SCIENTIFIC-COLLABORATION & 24 & 4 & 35 \\
\hline UNIVERSITY-LIBRARIES & 14 & 2 & 17 \\
\hline WEB-OF-SCIENCE & 13 & 2 & 20 \\
\hline SCIENTIFIC-JOURNALS & 12 & 2 & 19 \\
\hline HIGHER-EDUCATION & 9 & 4 & 20 \\
\hline SOCIAL-STRUCTURE & 5 & 2 & 9 \\
\hline
\end{tabular}

El tema Scientific-Production es un tema básico y transversal, poco desarrollado internamente pero bien relacionado con el resto de los temas de la revista. Es el que contiene mayor número de documentos del periodo y el segundo en citas. Es un tema nuevo que evoluciona del anterior de la 
Bibliometría. Al tratar sobre la producción científica abarca temas como las universidades, las tesis doctorales o las disertaciones propias del ámbito de las Ciencias Sociales, así como la investigación, en general, la productividad investigadora y la gestión del conocimiento.

El tema Web es básico y transversal; en el periodo anterior estaba en el cuadrante inferior izquierdo como un tema emergente; en este periodo ya aparece como un tema básico y transversal, con una densidad bastante baja, pero razonablemente bien conectado con otros temas de la revista, lo cual parece lógico debido a que la web, hoy en día, ya no es una novedad, es completamente transversal a la ciencia. Contiene elementos típicos como pueden ser la web 2.0 o Internet, pero también todo lo que tenga que ver con aspectos propios del área de la revista como las bibliotecas, la información o la educación.

Por último, creemos necesario aclarar de que trata el tema Estructura Social a pesar de que no sea un tema destacado, está relacionado con las temáticas que tienen que ver con las "redes sociales", enfocándose específicamente en la estructura de dichas redes sociales.

\subsubsection{Mapa de evolución conceptual}

Se ha desarrollado un análisis de los temas detectados en cada periodo de tiempo teniendo en cuenta las palabras clave y su evolución a lo largo del tiempo; para ello, se ha usado de nuevo SciMAT. De esta manera, se pudieron identificar las áreas temáticas que concentran la investigación que se ha realizado en REDC. Se han identificado dos grandes áreas temáticas: Bibliometría y Web.

La evolución conceptual y las áreas temáticas detectadas se muestran en la Figura 8. En este mapa las líneas continuas significan un nexo temático, una línea discontinua de puntos quiere decir que los temas vinculados comparten palabras clave diferentes al nombre de los temas, el grosor de la línea es proporcional al índice de inclusión, y el tamaño de la esfera es proporcional al número de documentos que alberga cada tema. También se ha diferenciado cada área temática por el sombreado. Por último, las medidas de rendimiento de las áreas temáticas detectadas se muestran en la Tabla VI.

El área temática Bibliometría es con mucho la más importante de la REDC y obtiene también los mejores indicadores de rendimiento. Tiene 7 veces más documentos que la segunda área temática y 11 veces más de citas. Tiene el mismo número de temas en el primer y en el segundo periodo. Además, está formada en su mayoría por temas moto-
Figura 8. Evolución temática de la REDC 20082018

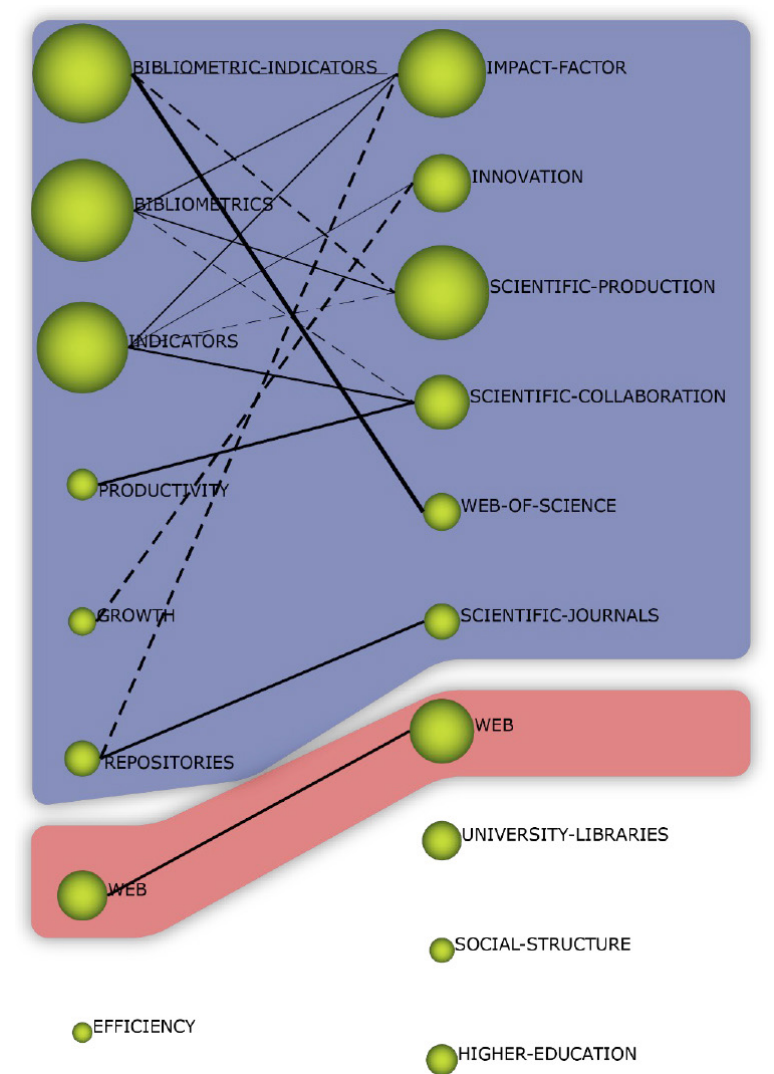

Tabla VI. Rendimiento de las áreas temáticas de la REDC

\begin{tabular}{|l|c|c|c|}
\hline $\begin{array}{l}\text { Área } \\
\text { temática }\end{array}$ & $\begin{array}{c}\text { Número de } \\
\text { documentos }\end{array}$ & $\begin{array}{c}\text { Número } \\
\text { de citas }\end{array}$ & Índice-H \\
\hline Bibliometría & 203 & 741 & 13 \\
\hline Web & 51 & 110 & 6 \\
\hline
\end{tabular}

res y básicos, relacionados con indicadores bibliométricos, indicadores de evaluación de la ciencia, Factor de Impacto, y todo lo que tenga que ver con asuntos científicos como son las revistas, repositorios, bases de datos como la Web-of-Science, colaboración científica y producción científica.

La Web es un área temática que cubre los distintos aspectos relacionados con la misma, como son Internet, el movimiento 2.0, las webs educativas o de bibliotecas, así como el tratamiento de la información en la misma. Además, toca temas relacionados con la usabilidad y la accesibilidad de la Web. Es curioso que esta área monotemática en el primer periodo está en una posición en medio de los temas motores y los básicos, y en el se- 
gundo periodo digamos que pierde esa fuerza para adentrarse en los temas básicos y transversales, entre otras cosas porque en el primer periodo tiene menos documentos, pero muchas más citas que en el segundo, donde tiene más documentos, pero bastantes menos citas.

\section{DISCUSIÓN}

A continuación, se van a poner en relación los resultados obtenidos en este estudio con lo que se había hecho previamente. Un reciente artículo sobre la internacionalización de la revista (PandiellaDominique y otros, 2019), también analiza las temáticas principales de la misma, destaca, como en este caso, la Bibliometría, seguida por las Bibliotecas, las revistas científicas y los contenidos digitales / sedes Web que sería equivalente a nuestra área temática Web. Con ligeras diferencias, las temáticas más destacadas son las mismas en ambos estudios.

El estudio de Guallar y otros (2017) también lleva a cabo un análisis temático de artículos publicados entre 2012-2014 en WoS y Scopus sobre esta área, con una codificación manual de los artículos en función del tema. De nuevo la temática con mayor proporción de artículos es la Bibliometría en esta disciplina, con un 19\% de los artículos de todas las revistas. Al ofrecer los resultados también individualizados por revistas, este porcentaje sube en la REDC al 33\%. No coincide con nuestro estudio en el que la segunda opción con mayor número de artículos en la REDC es Técnicas Auxiliares y otras disciplinas.

Terminando con los estudios sobre la propia revista, en la investigación sobre la historia de la REDC (Equipo Editorial REDC, 2012), se presenta una distribución temática de los artículos publicados por años, en el periodo 2006-2011, coincidiendo con el presente estudio, en primer lugar, aparecen los estudios Bibliométricos, y en segundo lugar, los Recursos Electrónicos, páginas Web, Internet, etc.

Queremos resaltar aquí también un estudio que realiza un análisis de co-palabras y de temáticas, teniendo en cuenta los artículos de autores de instituciones españolas en la WoS entre 1985 y 2014 en nuestra categoría (Olmeda-Gómez y otros, 2017). Destacan entre las temáticas la Web, todo lo relativo a la bibliometría, así como los libros electrónicos, gestión de derechos digitales o servicios de traducción. Como se puede observar las temáticas de la REDC se reflejan en este estudio, a la par que aparecen temáticas que no están muy presentes en la revista.

En cuanto al panorama internacional, es muy interesante un estudio realizado sobre la base de da- tos LISA (Gonzalez-Alcaide y otros, 2008), donde, en primer lugar, los estudios sobre la práctica bibliotecaria siguen siendo los más numerosos como ya lo eran en los años 60,70 y 80 , con una preeminencia clara de las bibliotecas universitarias. La mayor novedad con respecto a lo que ya se había dicho en estudios anteriores es la importancia obtenida por la Web, al igual que tienen un papel importante las publicaciones basadas en estudios bibliométricos sobre las ciencias de la salud. Como vemos, coincide con lo dicho en este estudio, salvo por la importancia preponderante de cada tema en cada uno de los casos.

\section{CONCLUSIONES}

En este trabajo se ha llevado a cabo un análisis bibliométrico de la Revista Española de Documentación Científica desde que se incluyó en la WoS en el año 2008 hasta el año 2018. Ha consistido en un análisis de rendimiento con los indicadores más importantes y un análisis temático a través de mapas de la ciencia. Se pueden sacar las siguientes conclusiones:

- La revista ha atraído el interés de la comunidad científica a lo largo de los años, lo que se observa por el crecimiento moderado pero paulatino de las citas y el índice de impacto.

- El Factor de Impacto de la REDC ha aumentado levemente a lo largo de los años, a pesar de ello, esto no le ha hecho consolidarse en el segundo cuartil en la categoría JCR "Information Science \& Library Science", sino que ha ido alternando en estos últimos 11 años entre el segundo y el tercer cuartil.

- Se han identificado los autores que han desempeñado un papel destacado en el desarrollo de la REDC. Algunos de ellos, los Drs. JiménezContreras, Torres-Salinas y Delgado-Lopez-Cózar, son los que más documentos y más citas han recibido entre el año 2008 y el 2018.

- Durante el periodo 2008-2018, España, Cuba y Colombia son los países más productivos, siendo España el más productivo, obteniendo más publicaciones que el resto de los países juntos. Los países de América Latina son los que más publican en la REDC por la facilidad del idioma. Estos datos coinciden con un estudio anterior sobre la REDC (Pandiella-Dominique y otros, 2019).

- Las publicaciones de la REDC se centran en dos grandes áreas temáticas: Bibliometría y Web. Aunque no formen parten de un área temática como tal. También se publican documentos sobre bibliotecas, en concreto sobre bibliotecas universitarias. 
- Hemos identificado un tema que ha atraído el interés de la comunidad científica y podría seguir haciéndolo: la Web of Science, incluida dentro del área temática de Bibliometría. Esta base de datos de información científica ha llamado la atención de los investigadores en el último periodo y es probable que sigan apareciendo artículos sobre esta temática en el futuro. Hay que matizar que en la REDC se han publicado escasos artículos sobre la WoS, sin embargo, se trata de la base de datos más utilizada para obtener los datos en los que se basan los estudios bibliométricos, lo cual motiva que los autores la incluyan como palabra clave en los artículos.

\section{REFERENCIAS}

Alonso, S.; Cabrerizo, F. J.; Herrera-Viedma, E.; Herrera, F. (2009). H-Index: A review focused in its variants, computation and standardization for different scientific fields. Journal of Informetrics, 3(4), 273-289. https:// doi.org/10.1016/j.joi.2009.04.001

Börner, K.; Chen, C.; Boyack, K. W. (2005). Visualizing knowledge domains. Annual Review of Information Science and Technology, 37(1), 179-255. https://doi. org/10.1002/aris.1440370106

Braun, T.; Glänzel, W.; Schubert, A. (2006). A Hirsch-type index for journals. Scientometrics, 69(1), 169-173. https://doi.org/10.1007/s11192-006-0147-4

Callon, M.; Courtial, J. P.; Turner, W. A.; Bauin, S. (1983). From translations to problematic networks: An introduction to co-word analysis. Social Science Information, 22(2), 191-235. https://doi. org/10.1177/053901883022002003

Cartes-Velásquez, R.; Manterola-Delgado, C. (2014). Bibliometric analysis of articles published in ISI dental journals, 2007-2011. Scientometrics, 98(3), 22232233. https://doi.org/10.1007/s11192-013-1173-7

Cobo, M. J; Martínez, M. A.; Gutiérrez-Salcedo, M.; Fujita, H.; Herrera-Viedma, E. (2015b). 25 years at Knowledge-Based Systems: A bibliometric analysis. Knowledge-Based Systems, 80, 3-13. https://doi. org/10.1016/j.knosys.2014.12.035

Cobo, M. J.; López-Herrera, A. G.; Herrera-Viedma, E.; Herrera, F. (2011a). An approach for detecting, quantifying, and visualizing the evolution of a research field: A practical application to the Fuzzy Sets Theory field. Journal of Informetrics, 5(1), 146-166. https://doi. org/10.1016/j.joi.2010.10.002

Cobo, M. J.; López-Herrera, A. G.; Herrera-Viedma, E.; Herrera, F. (2011b). Science mapping software tools: Review, analysis, and cooperative study among tools. Journal of the American Society for Information

\section{AGRADECIMIENTOS}

Este trabajo ha sido financiado por la Universidad de Granada, ayuda al Contrato Puente. Al proyecto TIN2016-75850-R "Sistemas inteligentes de toma de decisiones y consenso en ambiente difuso: Aplicaciones en e-salud y e-comercio".

\section{ACKNOWLEDGEMENTS}

This work has been financed by the University of Granada through the Contrato Puente. The authors would like to acknowledge FEDER funds under grants TIN2016-75850-R "Intelligent systems of decision making and consensus in diffuse environment: Applications in e-health and e-commerce".

Science and Technology, 62(7), 1382-1402. https:// doi.org/10.1002/asi.21525

Cobo, M. J.; López-Herrera, A. G.; Herrera-Viedma, E.; Herrera, F. (2012a). SciMAT: A new science mapping analysis software tool. Journal of the American Society for Information Science and Technology, 63(8), 16091630. https://doi.org/10.1002/asi.22688

Coulter, N.; Monarch, I.; Konda, S. (1998). Software engineering as seen through its research literature: A study in co-word analysis. Journal of the American Society for Information Science, 49(13), 1206-1223. https://doi.org/10.1002/(SICI)10974571(1998)49:13<1206: :AID-ASI7> 3.3.CO;2-6

De-Moya-Anegón, F.; Jiménez-Contreras, E. (1999). Autores españoles más citados en biblioteconomía y documentación. El Profesional de la Información, 8(5), 28-29.

Delgado-Lopez-Cozar, E. (2000). Diagnóstico de la investigación en Biblioteconomía y Documentación en España (1976-1996): estado embrionario. Revista de Investigación Iberoamericana En Ciencia de la Información y Documentación, 1(1), 79-93.

Equipo Editorial REDC. (2012). Historia de la Revista Española de Documentación Científica. Revista Española de Documentación Científica, 35(4), 521-521.

Ferran-Ferrer, N.; Guallar, J.; Abadal, E.; Server, A. (2017). Research methods and techniques in Spanish library and information science journals (2012-2014). Information Research, 22(1), 1-19.

Frías Montoya, J. A. (1996). La investigación en biblioteconomía y documentación en España a debate. Educación y Biblioteca., 8(73), 17-19.

Garfield, E. (1972). Citation Analysis as a Tool in Journal Evaluation: Journals can be ranked by frequency and impact of citations for science policy studies. Science, 178(4060), 471-479. https://doi.org/10.1126/science. 178.4060 .471 
Gonzalez-Alcaide, G.; Castello-Cogollos, L.; NavarroMolina, C.; Aleixandre-Benavent, R.; ValderrarnaZurian, J. C. (2008). Library and information science research areas: Analysis of journal articles in LISA. Journal of the American Society for Information Science and Technology. 59(1), 150-154. https://doi. org/10.1002/asi.20720

Guallar, J.; Ferran-Ferrer, N.; Abadal, E.; Server, A. (2017). Revistas científicas españolas de información y documentación: análisis temático y metodológico. El Profesional de la Información, 26(5), 947-960. https:// doi.org/10.3145/epi.2017.sep.16

Guan, J.; Gao, X. (2008). Comparison and evaluation of Chinese research performance in the field of bioinformatics. Scientometrics, 75(2), 357-379. https://doi. org/10.1007/s11192-007-1871-0

He, Q. (1999). Knowledge discovery through co-word analysis. Library Trends, 48(1), 133-159. Disponible en http://www.ideals.illinois.edu/bitstream/ handle/2142/8267/?sequence=1 [Fecha de consulta: 09/04/2019].

Hirsch, J. E. (2005). An index to quantify an individuals scientific research output, Proceedings of the National Academy of Sciences, 102. 16569-16572. https://doi. org/10.1073/pnas.0507655102

Huang, M. H; Chang, C. P. (2014). Detecting research fronts in OLED field using bibliographic coupling with sliding window. Scientometrics, 98(3), 1721-1744. https://doi.org/10.1007/s11192-013-1126-1

Jiménez-Hidalgo, S. (2007). Análisis de la autoría en la Revista Española de Documentación Científica (19972005). Revista Española de Documentación Científica, 30(3), 305-322. https://doi.org/10.3989/redc.2007. v30.i3. 387

Liu, G. Y.; Hu, J. M.; Wang, H. L. (2012). A co-word analysis of digital library field in China. Scientometrics, 91(1), 203-217. https://doi.org/10.1007/s11192011-0586-4

López-Robles, J. S,; Guallar, J.; Otegi-Olaso, J. R.; Gamboa-Rosales, N. K. (2019). El Profesional de la información (EPI): Bibliometric and thematic analysis (2006-2017). El Profesional de la Información, 28(4). https://doi.org/10.3145/epi.2019.jul.17

Martínez, M. A.; Herrera, M.; López-Gijón, J.; HerreraViedma, E. (2014). H-Classics: characterizing the concept of citation classics through $\mathrm{H}$-index. Scientometrics, 98(3), 1971-1983. https://doi.org/10.1007/ s11192-013-1155-9

Merigó J.M.; Miranda J.; Modak N.M.; Boustras G.; de la Sotta C. (2019). Forty years of Safety Science: A bibliometric overview. Safety Science, 115, https:// doi.org/10.1016/j.ssci.2019.01.029

Moed, H. F.; De Bruin, R. E.; Van Leeuwen, T. N. (1995). New bibliometric tools for the assessment of national research performance: Database description, overview of indicators and first applications. Scientometrics, 33(3), 381-422. https://doi.org/10.1007/BF02017338
Montero-Díaz, J.; Cobo, M. J.; Gutiérrez-Salcedo, M.; Segado-Boj, F.; Herrera-Viedma, E. (2018). A science mapping analysis of 'Communication' WoS subject category (1980-2013). Comunicar, 26(55), 81-91. https://doi.org/10.3916/C55-2018-08

Moral-Munoz, J. A.; Carballo-Costa, L.; Herrera-Viedma, E., Cobo, M.J. (2019). Production trends, collaboration, and main topics of the integrative and complementary oncology research area: a bibliometric analysis. Integrative Cancer Therapies, 18, 1-14. https://doi. org/10.1177/1534735419846401

Noyons, E. C. M.; Moed, H. F.; Luwel, M. (1999). Combining mapping and citation analysis for evaluative bibliometric purposes: A bibliometric study. Journal of the American Society for Information Science, 50(2), 115-131. https://doi.org/10.1002/(SICI)10974571(1999)50:2<115::AID-ASI3>3.0.CO;2-J

Olmeda-Gómez, M.; Ovalle-Perandones, A.; PerianesRodríguez, A. (2017). Co-word analysis and thematic landscapes in Spanish information science literatura, 1985-2014. Scientometrics. 113(1), 195-217. https:// doi.org/10.1007/s11192-017-2486-8

Pandiella-Dominique, A.; García-Zorita, C. ; Sanz-Casado, E. (2019). Análisis de la internacionalización de la Revista Española de Documentación Científica: 2010-2015. Revista Española de Documentación Científica, 42(1), e223. https://doi.org/10.3989/redc.2019.1.1594

Pérez-Alvarez-Ossorio, J. R. (1997). Cobertura temática y procedencia institucional de los artículos publicados en la Revista Española de Documentación Científica en sus veinte años de existencia. Revista Española de Documentación Científica, 20(3), 290-298. https://doi. org/10.3989/redc.1997.v20.i3.584

Raeeszadeh, M.; Karamali, M.; Sohrabi, A. (2018). Science mapping of "Trauma Surgery" by co-word analysis and thematic clustering in MEDLINE. Archives of Trauma Research, 7(3), 102-108. https://doi.org/10.4103/ ATR.ATR_48_18

Ríos-Hilario, A. B.; Travieso-Rodríguez, C. (2013). Estudio de la Revista Española de Documentación Científica: análisis comparativo de los periodos 1989-1999 y 2000-2010. Revista Española de Documentación Científica, 36(3), e016. https://doi.org/10.3989/ redc.2013.3.990

Ríos-Hilario, A. B. (2001). Diez años de investigación en la Revista Española de Documentación Científica (19891999). Revista Española de Documentación Científica, 24(4), 433-449. https://doi.org/10.3989/redc.2001. v24.i4.70

Schubert, A. (2007). Successive h-indices. Scientometrics, 70(1), 201-205. https://doi.org/10.1007/ s11192-007-0112-x

Sternitzke, C.; Bergmann, I. (2009). Similarity measures for document mapping: A comparative study on the level of an individual scientist. Scientometrics, 78(1), 113-130. https://doi.org/10.1007/s11192007-1961-z 
Tang, L.; Shapira, P. (2011). China-US scientific collaboration in nanotechnology: patterns and dynamics. Scientometrics, 88(1), 1-16. https://doi.org/10.1007/ s11192-011-0376-z

Torres-Salinas, D.; Jiménez-Contreras, E.; RobinsonGarcía, N. (2014). Tendencias en mapas de la ciencia: co-uso de información científica como reflejo de los intereses de los investigadores. El Profesional de la Informacion, 23(3), 253-258. https://doi.org/10.3145/ epi.2014.may.05

Van-Raan, A. F. J. (2004). Measuring Science. En Handbook of Quantitative Science and Technology Research, pp. 19-50. Dordrecht: Springer Netherlands. https://doi. org/10.1007/1-4020-2755-9_2 